\title{
The influence of fatty acid composition on glycaemic response and satiety
}

\author{
M. E. Clegg, M. Pratt and C. J. Henry \\ Functional Food Centre, School of Life Sciences, Oxford Brookes University, Gipsy Lane, Oxford, OX3 OBP, UK
}

The concept of glycaemic response is now well established as a tool to help in the management of many widespread chronic health conditions $^{(1)}$. A variety of studies have shown that fat can reduce glycaemic response ${ }^{(2)}$. Although medium chain TAG (MCT) have been implicated in improved insulin sensitivity, no research to date has examined its effect on glycaemic response ${ }^{(3)}$. The aim of this study was to compare the glycaemic response and satiety of pancakes containing either sunflower oil, olive oil, butter, MCT oil or a control containing no oil.

Ten healthy volunteers (9f $2 \mathrm{~m} ; 27.7$ (SD 5.5) years; 1.68 (SD 0.09) m; 61.2 (SD 9.3) kg) were tested on five occasions following an overnight fast. Volunteers were fed pancakes containing $50 \mathrm{~g}$ of available carbohydrate and $845.16 \mathrm{~kJ}$ of sunflower oil, olive oil, butter, MCT oil or a control containing no oil. Baseline and postprandial glycaemic response was measured using finger prick blood samples and satiety was measured using visual analogue scales (VAS). Analysis was completed using repeated measures ANOVA. Statistical significance was set at $P<0.05$, data is expressed as mean (SD).

There were significant differences in glycaemic response between the different pancakes $(P=0.040)$. The predominant differences existed between the control and olive oil $(P=0.004)$ and between olive and butter $(P=0.037)$. Olive oil had the lowest GR and control the highest. There were also significant differences in the VAS parameters fullness $(P=0.022)$ and amount $(P=0.027)$ but not hunger or desire to eat $(P>0.05)$. The main differences for VAS amount occurred between the MCT and both sunflower $(P=0.028)$ and control $(P=0.010)$ and for VAS fullness between control and MCT $(P=0.023)$, sunflower $(P=0.02)$ and olive oil $(P=0.05)$. MCT had the greatest satiety scores and control the lowest.

\begin{tabular}{|c|c|c|c|c|c|c|c|c|c|c|}
\hline & \multicolumn{2}{|c|}{ Sunflower oil } & \multicolumn{2}{|c|}{ Olive oil } & \multicolumn{2}{|c|}{ Butter } & \multicolumn{2}{|c|}{ MCT oil } & \multicolumn{2}{|c|}{ Control } \\
\hline & Mean & $\mathrm{SD}$ & Mean & SD & Mean & $\mathrm{SD}$ & Mean & SD & Mean & $\mathrm{SD}$ \\
\hline $\begin{array}{l}\text { Glycaemic } \\
\text { response* }\end{array}$ & 47.8 & 39.9 & 36.5 & 21.3 & 60.7 & 39.4 & 47.6 & 42.7 & 76.4 & $\overline{43.8}$ \\
\hline Hunger & 44.3 & 5.9 & 47.5 & 10.1 & 47.5 & 13.1 & 46.6 & 8.0 & 54.6 & 13.6 \\
\hline Desire to eat & 45.6 & 8.1 & 46.2 & 9.6 & 49.6 & 13.2 & 46.9 & 7.2 & 54.6 & 14.9 \\
\hline Fullness* & 41.1 & 13.3 & 39.6 & 12.3 & 37.9 & 11.4 & 41.2 & 11.9 & 30.5 & 8.2 \\
\hline Amount* & 39.2 & 13.3 & 40.0 & 15.6 & 39.5 & 13.0 & 44.7 & 14.8 & 32.3 & 10.3 \\
\hline
\end{tabular}

Glycaemic response and satiety following each of the pancake breakfasts. $* P<0.05$.

This data indicates that MCT has the ability to increase satiety but not significantly influence glycaemic response. This is in keeping with previous data indicating that MCT can be used as a weight management tool ${ }^{(4)}$.

1. Jenkins DJ, Wolever TM, Taylor RH et al. (1981) Am J Clin Nutr 34, 362-366.

2. Henry CJ, Lightowler HJ, Newens KJ et al. (2008) Int J Food Sci Nutr 59, 61-69.

3. Flatt JP, Ravussin E, Acheson KJ et al. (1985) J Clin Invest 76, 1019-1024.

4. Clegg ME (2010) Int J Food Sci Nutr 61, 653-679. 